\title{
An Approach to Implement Cost Efficient Space Detection Technology with Lower Complexity for Smart Parking System
}

\author{
Himal Pratap Singh ${ }^{\star 1}$, Om Prakash Uniyal ${ }^{2}$, Kireet Joshi ${ }^{3}$ \\ Graphic Era University (GEU), Dehradun \\ *Corresponding author, e-mail: himalpratap@gmail.com¹, om.uniyal19@gmail.com², \\ joshikireet@gmail.com ${ }^{3}$
}

\begin{abstract}
As the population of world is growing day by day, there is a rapid increment in the vehicle production. With the increase in number of the vehicles, more parking spaces and efficient ways of parking are required. In the proposed system, a new and cost efficient way of finding the free spaces in parking lot is proposed and also different space detection technologies are compared. This system will be helpful for the drivers in finding the vacant spaces in the car parking lots using a simple technology in which a balloon will be used to determine the vacant parking spaces. Since this technology is cost efficient and effective,this system is termed as smart parking system.
\end{abstract}

Keywords: space detection, cost efficiency, ultrasonic sensors, CC TV, balloon

Copyright $\odot 2015$ Institute of Advanced Engineering and Science. All rights reserved.

\section{Introduction}

Human life depends very much upon two factors that are time and cost. Quality of life is increasing day by day that is why number of people inhabiting urban life is increasing. There is a significant role of centralized public facilities in urban life. Shopping complexes are the main attraction both for a city's inhabitant as well as for a visitor. With the rise of shopping complexes that are providing a variety of modern facilities, more and more people want to visit them. Therefore more shop owners prefer to locate their shops in these shopping complexes to attract more customers [1].

Gone are the days when pure selling and buying were used to take place in a shopping complex. Now, they have begun providing many more services like banking, food courts, cinema halls and children's play areas et cetera. These things have influenced shopping culture and behavior [2].

Availability of sufficient parking space for the visitors is one of the main issues in developing these shopping complexes. Offering sufficient number of safe and secure parking spaces is one of the few factors that can increase customer loyalty and attract customers to visit a shopping complex more frequently. There are several types of parking lots such as multilevel parking, barrier gate parking, roadside parking etc. The factors by which patrons choose a specific parking lot are safety, weather conditions and car parking fees respectively [3].

The objectives of this paper are to highlight parking lots' importance, indicate the difficulty that the drivers have in parking their vehicles and to propose an applicable and cost effective solution to solve these problems.

The introduction section of this paper details the importance of shopping complexes and parking lots. In part 2, the current parking lot problems and the difficulties that customers face in parking lots are discussed. Part 3 discusses the proposed technology to detect the parking space and compares it with several other technologies that are used to find the vacant parking space. Section 4 provides a system overview and explains the feature of the technology that is going to be proposed. Section 5 explains the system architecture and the things that is required to implement it and the last section offers conclusion and explains the current research. 


\section{Statement of Parking Lot Problems \\ 2.1. Hardships in Finding Vacant Spaces}

Finding a vacant space quickly is difficult in multilevel parking lots, especially on weekends or public holidays. During weekends or public holidays,finding vacant space can take a plenty of time. Stadiums or shopping complexes are crowded at peak periods and difficulty in finding vacant space at these places is a big problem for customers [4]. Insufficient car park spaces can lead to traffic congestion and driver frustration [5].

\subsection{Improper Parking}

If a car is parked in such a way that it occupies more than one parking slots rather than one, this is called improper parking. Improper parking can happen when a driver is not careful about the problems that other people may face due to his way of parking. Sometimes improper parking occurs when a driver parks a bit outside of the lines of a parking space. The driver may notice his improper parking after leaving his car, but he may not be willing to unlock his car, restart it, and adjust it to be parked accurately. This matter may annoy other drivers and most of the time a driver who wants to park in a small leftover slot will give up and feel frustrated.

Figure 1 presents an improper parking situation.

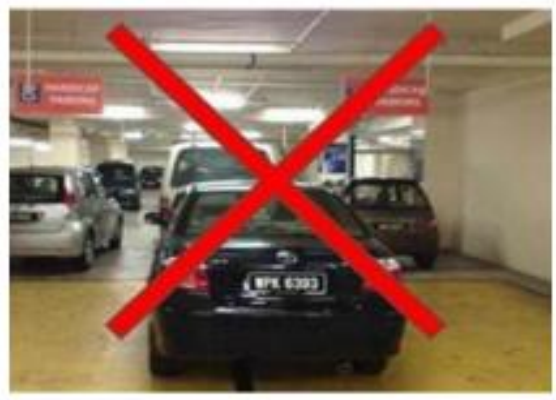

Figure 1. Improper Parking [14]

\subsection{Parking Fee Payment}

Parking fee payment sometimes become a time consuming activity for people. Since many current payment machines just accept small notes and coins, therefore finding the exact amount and queuing for payment is not pleasant for drivers. So, providing services that make payment convenient are required. One survey showed that queuing up for payment and finding coins or small notes for parking fee payment is troublesome. Moreover, most respondents agreed that using Touch ' $n$ ' Go (a system that allows simply swiping a card and deducts fees from inside credit) is useful and will decrease queuing up time [3].

\section{Detection Technology Review}

The choice of appropriate detection technique depends on the objectives and scope of the project [6]. Two types of detection technology, vision-based and sensor-based, are discussed in this study. Vision-based detection tachnology uses closed-circuit television (CCTV) - usually one camera is responsible for more than one parking space - and an image processing software to detect parking space status. Sensor-based detection methods use one sensor for each individual parking space.

\subsection{Vision-Based Methods}

Monitoring parking lot vacancy is a significant technology which can be used for guiding cars to vacant spaces and for efficient use of the available parking spaces. Monitoring detection technology is divided into two categories. The first estimates the total number of remaining vacant spaces for the entire parking lot by counting incoming and outgoing vehicles. The second monitors the status of each individual space and it can be used to guide a car to a vacant space. To help drivers find the vacant parking space without much effort, intelligent parking systems should provide the specific location of vacant spaces and not just the total number of parking spaces [7]. To detect the status of an individual parking space, different 
methods have been utilized such as ultrasonic sensors placed at each space (it requires many sensors) or surveillance cameras placed at a high position (allowing supervision of a wide area by a few cameras, which is more useful in outdoor parking lots) [6]. CCTV cameras are generally used to track the positions of vehicles [8]. Detection technologies based on cameras and image processing suffer from a lack of accuracy and can be affected by environmental or weather circumstances. Major problems of vision based parking detection systems include shadow effects, occlusion effects, vacillation of lighting conditions, perspective distortion and eye sight of the driver. Light-colored cars in strong sunlight might mislead detector software into detecting a full space as a vacant space; in the same way, a shadowed area may be identified as a dark-colored vehicle by mistake, which leads an empty space to be mistakenly seen as occupied. When the sun is blocked by clouds, the change in lighting may also affect detection performance [7]. Variable light intensity is one of the biggest challenges in a camera-based detection system.

In this paper, the technology that is used is also a vision based detection technology. In that tehnology, a balloon filled with nitrogen is used to determine the vacant parking space.

\subsection{Sensor-Based Methods}

Another detection technology uses sensors to detect vacant parking spaces in a parking lot. With the availability of different types of sensors, selecting a suitable detection system is an important part of implementing a smart parking system. Many different factors play a role in choosing the proper sensor, including size, reliability, adaptation to environmental changes, robustness and cost [9].

Sensor technologies are categorized as intrusive and non-intrusive. Intrusive sensors need to be installed directly on the pavement surface; because of this digging and tunneling under the road surface are required. Magnetometers, pneumatic tubes, inductive loops, weightin-motion sensors and piezoelectric cables are considered to be intrusive sensors. Non-intrusive sensors only require fixing on the ceiling or on the ground of the parking lot. Ultrasonic sensors are categorized as non-intrusive sensors which mean that they require simpler installation compared to intrusive sensors [10]. Ultrasonic sensors can be used to realize small distance measured [11]. Ultrasonic sensors transmit the sound waves between $25 \mathrm{kHz}$ and $50 \mathrm{kHz}$. They use the reflected energy in analyzing and detecting the status of a parking space. Ultrasonic waves are emitted from the head of an ultrasonic vehicle detection sensor every 60 milliseconds and the presence or absence of vehicles is determined by the time differences between the emitted and received signals. Ultrasonic sensors can be used for counting thr number of vehicles and assessing the occupancy status of each parking space [12]. Ultrasonic sensors do have some disadvantages, particularly sensitivity to temperature changes and extreme air turbulence. Figure 2 shows how ultrasonic sensors work.

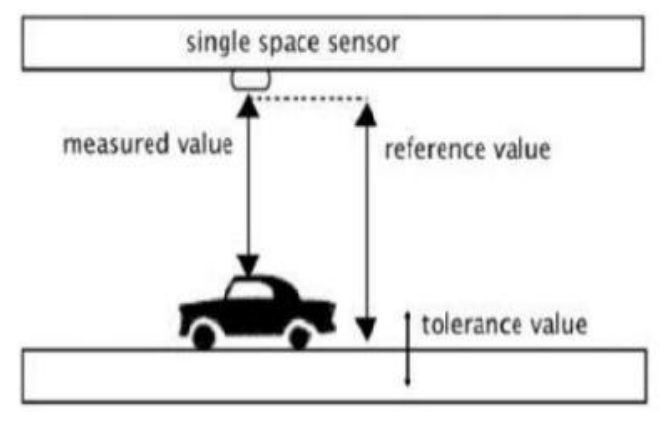

Figure 2. How an Ultrasonic Sensor Works [13]

\section{Description of Smart Parking System \\ 4.1. Smart Parking System User Overview}

In order to find vacant spaces, drivers look above the parking spaces from the gate of the parking lot to see whether, there a balloon is visible slightly above the parking space or not. If there is a balloon visible above the parking space, it means that particular parking space is 
vacant and, if there is no balloon visible above the parking space, it means that parking space is occupied. After entering the parking lot, drivers look at internal signs hanging from the ceiling at the end of each aisle. Each internal sign shows two parts: the number of available parking spaces and the direction (left, right or forward) of the aisle which has a vacant space.

\subsection{Smart Parking System Technical Overview}

The proposed Smart Parking Space detection system is based on balloon technology. To develop the Smart Parking System architecture, several pieces of equipment are required: balloons filled with nitrogen, strong yarn and pulleys.

For each individual car park, this would require one balloon to be fixed on each parking space as per given instructions. Balloon, will have nitrogen filled inside it which will allow it to float in the air. The balloon will be tied with a strong yarn. One side of the yarn will be tied to a screw at one side of the parking space followed with a pulley at the other side of the parking space as shown in the figure given below.

When the car will enter the parking space, it will pull the yarn with it and thus the level of the balloon will get lower which will indicate that the parking space is occupied.

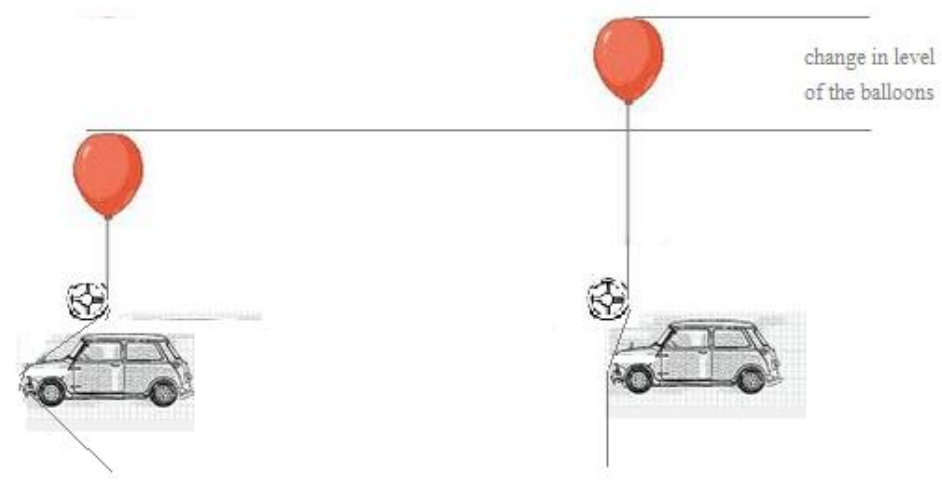

(a)

(b)

Figure 3. Working of the space detection technology

Figure 3(a) shows a car entering the parking space and the position of the balloon when car is entering. Figure 3(b) shows the parked car and the position of the balloon after the car is parked. Lines above the balloons show the change in the level of the balloon before and after the car is parked.

For improper parking detection, two extra sensors for each parking space, oriented horizontally along the lines demarcating the left and right boundaries of the space will be used. If any car is parked on the line, the sensor will trigger an alarm and then the driver should adjust his car within the lines until the beeping sound stops. Although some parking lots are using ultrasonic sensors and LED indicators at present time, improper parking systems are not in place. Figure 4 presents how SPS's line detection feature works.

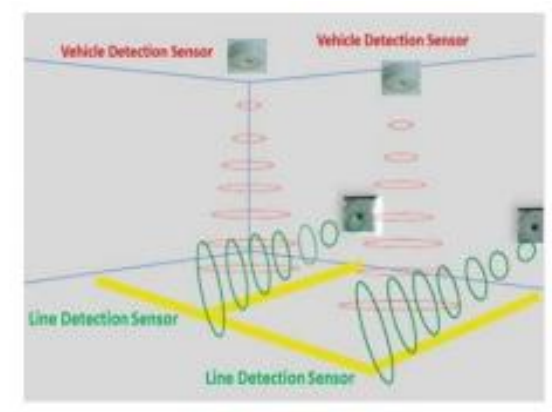

Figure 4. SPS Line Detection System Demo [14]

TELKOMNIKA Vol. 15, No. 3, September $2015: 415-419$ 


\section{Result \& Comparison}

After implementing the balloon based spaced detection system, it is found that this system can be installed in a very low cost. Camera based and ultrasonic sensor based space detection systems requires a lot of money to be installed comparatively. In addition to money factor, the implementation of ballon based space detection system is less complex and less time consuming. Apart from it, it requires very low maintenance. Based on these factors, the comparision of balloon based space detection system with the other systems described in this study is as follow:

\begin{tabular}{llll}
\hline DETECTION SYSTEM NAME & Camera Based & Ultrasonic Sensor Based & Ballon Based \\
\hline INSTALLATION COST & High & High & Very Low \\
COMPLEXITY & High & High & Negligible \\
MAINTENANCE & High & High & Low \\
EFFICIENCY & Good & Good & Good \\
\hline
\end{tabular}

\section{Conclusion}

The main contribution of this paper is to introduce the most significant parking problem i.e., finding an empty space and propose a solution. Balloon technology can be used for parking space detection and ultrasonic sensors can be used for improper parking detection. The proposed parking detection system would decrease searching time for vacant spaces and reduce instances of single cars improperly parking across two spaces. This parking space detection system can be considered the cheapest space detection system till date.

\section{Acknowledgements}

The authors would like to acknowledge the support of Graphic Era University for this research and would like to convey a special thanks to Mr. Devesh Pratap Singh for his valuable guidance. The authors would also like to thank Mr. Himanshu Joshi and Mr. Harish Singhania for their assistance.

\section{References}

[1] M Fishbein, I Ajzen. Belief, Attitude, Intention and Behaviour: An Introduction to Theory and Research. 1975.

[2] PWH Coopers. From Beijing to Budapest - Winning Brands. Winning Formats. 2005: 126.

[3] A Kianpisheh, N Mustaffa, JMY See, P Keikhosrokiani. User Behavioral Intention toward Using Smart Parking System. Proceeding of ICIEIS. Kuala Lumpur, Malaysia. 2011: 732-747.

[4] DBL Bong, KC Ting, KC Lai. Integrated Approach in the Design of Car Park Occupancy Information System (COINS). IAENG International Journal of Computer Science, IJCS. 2008; 35(1).

[5] MYI Idris, EM Tamil, NM Noor, KW Fong. Parking Guidance System Utilizing Wireless Sensor Network and Ultrasonic Sensor. Information Technology Journal. 2009.

[6] K Yamada, M Mizuno. A Vehicle Parking Detection Method Using Image Segmentation. Electronics and Communications in Japan, Part III: Fundamental Electronic Science (English translation of Denshi Tsushin Gakkai Ronbunshi). 2001; 84: 25-34.

[7] CC Huang, SJ Wang. A Hierarchical Bayesian Generation Framework for Vacant Parking Space Detection. Circuits and Systems for Video Technology, IEEE Transactions. 2001: 1.

[8] Fei Teng, Qing Liu. Robust Multiple Ship Tracking in Inland Waterway CCTV System. TELKOMNIKA Indonesian Journal of Electrical Engineering. 2014; 12(11): 7772-7777.

[9] I Masaki. Machine-vision Systems for Intelligent Transportation Systems. Intelligent Systems and their Applications, IEEE. 1998; 13: 24-31.

[10] J Scheeling, Car Park Monitoring System. University of Queensland. 2002.

[11] Dai Juan, Zhao Zhihong, Yan Minglian. Ultrasonic Automatic Tracking System. TELKOMNIKA Indonesian Journal of Electrical Engineering. 2014; 12(6): 4664-4670.

[12] LEY Mimbela, LA Klein. A Summary of Vehicle Detection and Surveillance Technologies used in Intelligent Transportation Systems. Southwest Technology Development Institute (SWTDI) at New Mexico State University (NMSU). 2000.

[13] Parking Consultants International. Parking Guidance Systems. Sydney: Parking Consultants International. 2009

[14] Amin Kianpisheh, Norlia Mustaffa, Pakapan Limtrairut, Pantea Keikhosrokiani. Smart Parking System (SPS) Architecture Using Ultrasonic Detector. International Journal of Software Engineering and Its Applications. 2012; 6(3). 